\title{
Carbon-13 Characterization and Modelling for Temperature Measurement-Based Proton Frequency
}

\author{
Monis Abdulmanan Abdullah ${ }^{1, *}$, Thar M. Badri Albarody ${ }^{1, \dagger}$, Puteri Sri M. Bt Megat \\ Yusoff ${ }^{1}$ and Alaa $^{1}$ Raad Hussein \\ ${ }^{1}$ Department of mechanical engineering, Universiti Teknologi PETRONAS, Malaysia
}

\begin{abstract}
The physical substance at high energy level with specific circumstances; tend to behave harsh and complicated, meanwhile, sustaining equilibrium or non-equilibrium thermodynamic of the system. Measurement of the temperature by ordinary techniques in these cases is not applicable at all. Likewise, there is a need to apply mathematical models in numerous critical applications to measure the temperature accurately at an atomic level of the matter. Those mathematical models follow statistical rules with different distribution approaches of quantities energy of the system. However, these approaches have functional effects at microscopic and macroscopic levels of that system. Therefore, this research study represents an innovative of a wireless temperature sensor, which utilizes proton resonance frequency of carbon-13 isotope material. In addition to that, this study also addresses the energy distribution of the particles by selecting an updated appropriate approach that has interesting points of limitation in the number of degree of freedom: (1) thermodynamically limits and (2) theoretical statistical thermodynamics observations. Lastly, the main idea of this paper is to visualize the analysis of temperate in the nanoscale system via statistical thermodynamics approach along with the material characterization of carbon-13 isotope.
\end{abstract}

\section{Introduction}

Temperature is the key concept of many engineering applications which it is directly related to the entropy and the energy of that system. The crystal-clear understanding of the temperature concept of a matter under high-temperature and harsh environments is a big challenge. High-temperature definition differs from one group of applications to another, firmly: the biochemistry science which deals with processes and substances that happen within living organisms, that may use the term high-temperature for $50^{\circ} \mathrm{C}$ and a material engineering for $2000^{\circ} \mathrm{C}$ [1]. In general, there are three core methods of measure the temperature. The first method is the direct contact i.e. by conventional devices, the second method is by radiation spectroscopic analysis, and the last method is by speculating the

\footnotetext{
* Corresponding author: monis_g03669@utp.edu.my

† Corresponding author: dher.albarody@utp.edu.my
} 
temperature from chemical composition [2]. In any of above-mentioned methods of measuring the temperature, the accuracy of interpretation of the temperature of any system is indeed based on a mathematical model with a list of assumptions ensemble within that system. In the nanoscale system, the interpretation of temperature is very difficult comparatively with a classical system due to the quantum theory applied for the atomic-level and based on the uncertainty phenomenon. In addition, the random probability distribution of mathematical tools, the generalized solution of the differential equation and complexity of the validate assumptions in terms of finite size or finite time. However, the statistical approach is an effective adoption to start with a generalized relation between the entropic form of a system and the probability distribution of the microstates of that system. Moreover, the statistical approach in terms of quantum mechanics provides precise details of the mechanical information of the system and comprehensive canonical energy distribution of it [3]. The temperature dependence on proton resonance frequency is a unique thermometry technique that has been used in many applications [4]. The spin-temperature theory has been verified and used for deep cooling over magnetization system in semiconductor structures [5]. Predicting molecular properties from the temperature of a sample by nuclear magnetic resonance has been studied in fields such as pharmacology, foods industry and many other applications [6]. Determining the sample temperature utilizing nuclear magnetic resonance used in eutectic temperature of metals during production has also been studied [7]. Furthermore, nuclear magnetic resonance has been studied for applying in the material at high temperature for many applications such as liquid metals, ionic conductors, and molten salts [8]. In this current work, introductory of new precise temperature sensor-based proton resonance frequency had been demonstrated.

\section{The statistical interpretation of the temperature}

The thermal equilibrium state of any system is not a static state because there are internal energy and motion related to that energy from the microscopical point of view of that system. In the microstate of a system, the equity relation reflects the thermal equilibrium between its macrostate based on transitivity, reflexivity and symmetric properties. The temperature is defined as an intensive variable of the thermal macrostate of a system that remains same for its microstate [9]. The maximum entropy of a system is defined by the thermal equilibrium state. Whereas in statistical approach, the entropy is a function of microstate probabilities, therefore, it is a variational problem. The variational problem can be handled effectively by the Lagrange multiplier [10].

$$
\begin{gathered}
S=k_{B} \mathcal{N}\left[(\ln \mathcal{N}-1)\left(1-\sum_{i} p_{i}\right)+\sum_{i} \sigma\left(p_{i}\right)\right] \\
\sum_{i} L\left(E_{i}\right)=\sum_{i} \mathcal{N}_{i} L\left(\mathcal{E}_{i}\right)=L(E) \\
\sum_{i} \mathcal{N}_{i} L_{N}\left(n_{i}\right)=L_{N}(N) \\
\sum_{i} \mathcal{N}_{i} L_{V}\left(v_{i}\right)=L_{v}(V)
\end{gathered}
$$




$$
\begin{gathered}
\sum_{i} N_{i}=\sum_{i} e^{-L\left(\varepsilon_{i}\right) / k_{B} T} \\
\sum_{i} \mathcal{N}_{i}=\mathcal{N} \\
p_{i}=\frac{\mathcal{N}_{i}}{\mathcal{N}}
\end{gathered}
$$

Whereas, $S$ represents the entropy, $k_{B}$ Boltzmann constant, $\mathcal{N}$ overall number of states, $p_{i}$ thermodynamic probability distribution of the macrostate, $\sigma$ general functional form, $\mathcal{E}_{i}$ energy level, $\mathcal{N}_{i}$ degeneracy number of the state, $E$ the energy, $N$ the number of particle and $V$ the volume. Equation (6) and (7), represent partition sum and probability distribution respectively [11]. In statistical thermodynamic approach, there are two types of particles, distinguishable and indistinguishable. If the macroscopic state of the system changes when particles exchange their positions, velocities and energy state; then, the particles are defined distinguishable [12]. Furthermore, if the assembly is crystal, the molecules can be categorized according to the positions they occupy, and it can be considered distinguishable in the crystal lattice. The general fact is stated in a study that there are two different classes of particles that are found, namely, fermions and bosons [13]. Fermions have half-integral spin angular momentum and obey the Pauli exclusion principle whereas electrons are considered as Fermi particle [14]. The heat takes place in the microscopic of a system depending on the position of the Fermi level i.e. the unpaired electrons excitation energy state in the last subshell of the atom [15]. The monatomic isotope carbon-13 (C-13) obeyed to the above-mentioned quantum considerations, which it is the selected material for this temperature sensor.

\section{Temperature measurement-based proton frequency}

The material applicable to the nuclear magnetic resonance concept has an odd isotope number, with the effect of the intensity of the magnetic field that can obtain the resonance frequencies of that material $[16,17]$. Less number of electron density of the material, the nucleus sensations are more to the external magnetic field; therefore, it is said to be deshielded and vice versa $[18,19]$. The de-shielded effected by the temperature is explained as an increase of temperature, increases the de-shielded; leading to downfield shifts of the chemical shift (the resonant frequency of a nucleus relative to a standard in a magnetic field) in the nuclear magnetic spectroscopy. Because the proton experiences higher external magnetic field, it needs a higher frequency to achieve resonance. Make an advantage of $\mathrm{C}$ 13 in terms of standardized material for NMR theory, stable material non-radioactive and high melting point up to $3350^{\circ} \mathrm{C}$ with a unique isotope signature, as well as carbon can be found in many application [20]. 


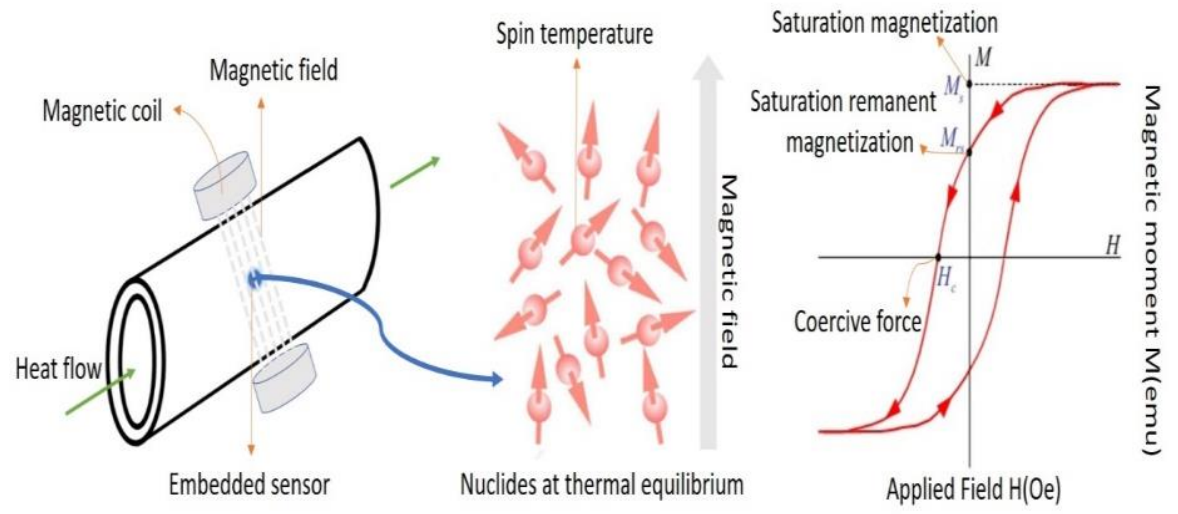

Fig. 1. Sensor working principle.

Figure 1 illustrates the working principle of the supposed wireless temperature sensor. In a thermal system, as shown, the sensor i.e. isotope carbon-13 is embedded in the structure whereas, it is at thermal equilibrium state with the system and subjected to a magnetic field. Nuclides of the $\mathrm{C}-13$, when the protons inside the nuclides subjected to a magnetic field a precession with phase coherence takes place which is known as spin-lattice relaxation or longitudinal relaxation time $\left(\mathrm{T}_{1}\right)$. Calculation of saturation magnetization (C-13 cannot increase its magnetization with an increase in the external applied magnetic field) and saturation remnant magnetization (measurement of the remaining magnetization when the external magnetic field dropped to zero) at thermal equilibrium of the system. In addition, this occurs in terms of time which can provide a precise temperature measurement of the thermal system.

\section{Carbon-13 morphology and microstructure}

\subsection{Powder x-ray diffractometer}

X-ray diffraction (XRD) characterization was performed with a Powder X-Ray Diffractometer (XRD, Model: $\mathrm{X}^{\prime}$ Pert3 Powder \& Empyrean, PANalytical). It provides preliminary information about $\mathrm{C}-13$ sample nature, which guides to a hypothesis about sample structure [21]. Inter-plane spacing can be calculated, crystal directions and the atomic packing factor; which it is related to the volume of atoms and volume of the unit cell are also computed $[22,23]$. Those are obtained by the atomic radius; that is applied to diffraction planes for the diffraction peaks to achieve a first quantitative understanding of the crystallographic structure as shown in figure 2. 


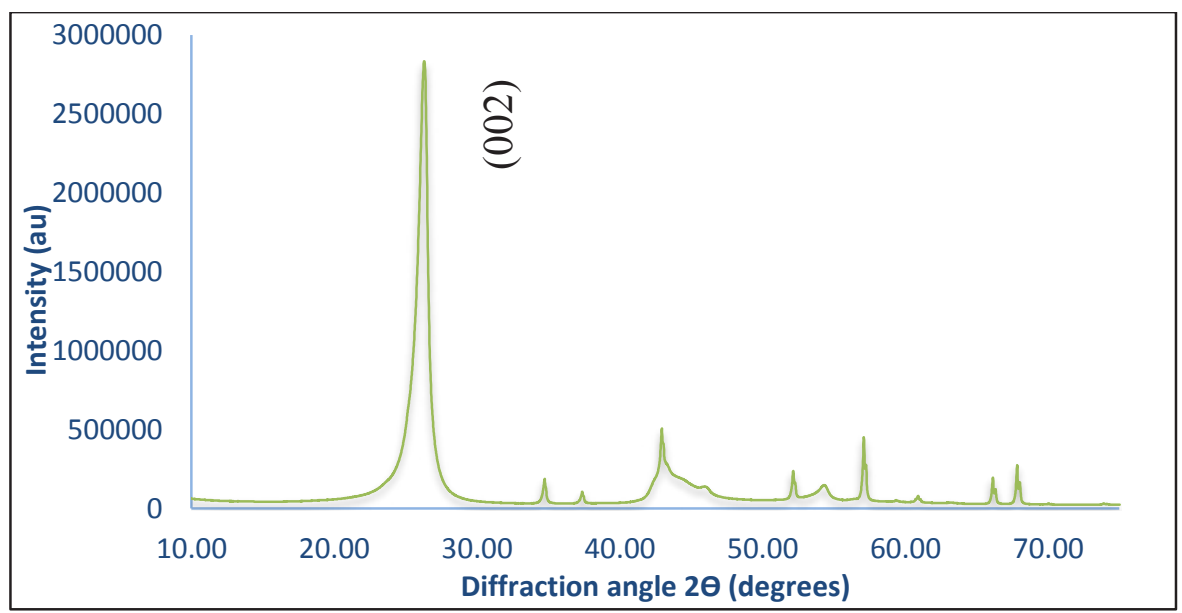

Fig. 2. XRD of pure nano Carbon-13.

\subsection{X-ray photoelectron spectrometer}

The Thermo Scientific K-Alpha XPS machine set with the high-resolution spectrometer was used for the analysis. Physical features and chemical state analysis of the C-13, spin orbital splitting, and peak area ratios of the sample has been examined with XPS, which assists in element identification [24]. In the base of binding energy by check peak positions, it was recorded as 284.68(eV). Based on the energy resolution of the XPS instrument used, slight variation in binding energy $<0.35 \mathrm{eV}$ is considered as insignificant. The relative peak intensities with spin-orbital splitting and area ratios for assisting peak identification has been yielded, as shown in figure 3 .

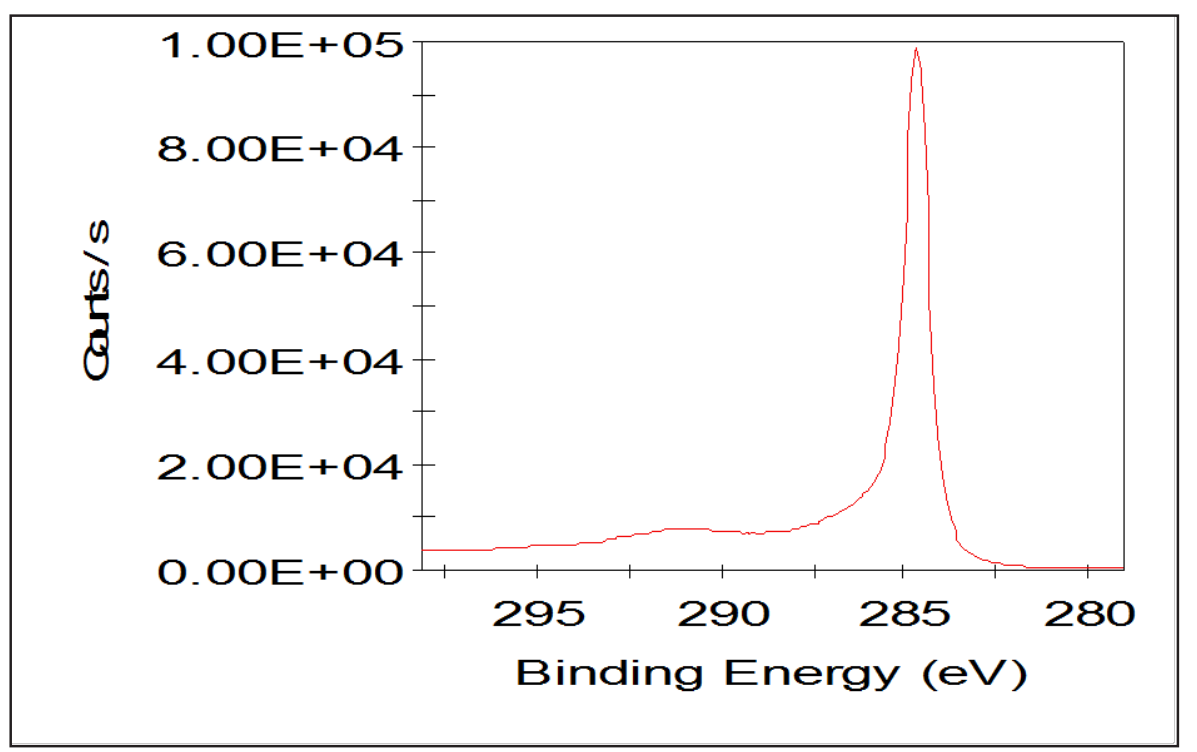

Fig. 3. XPS of pure nano Carbon-13. 
Particles morphology of our samples was investigated using Field-Emission Scanning Electron Microscope (FESEM)/Energy Dispersive X-Ray Model: Zeiss Supra 55 VP. It provides an information of the material sample of $\mathrm{C}-13$ due to the atomic number of the sample as well as observation of topographic information of the material sample[25]. Measurement function allows dimensional and angle measurement of a specific point of interest on the SEM image. Additional to that, the guarantee of the material purity can be done through the mapping analysis as shown in Figure 4.
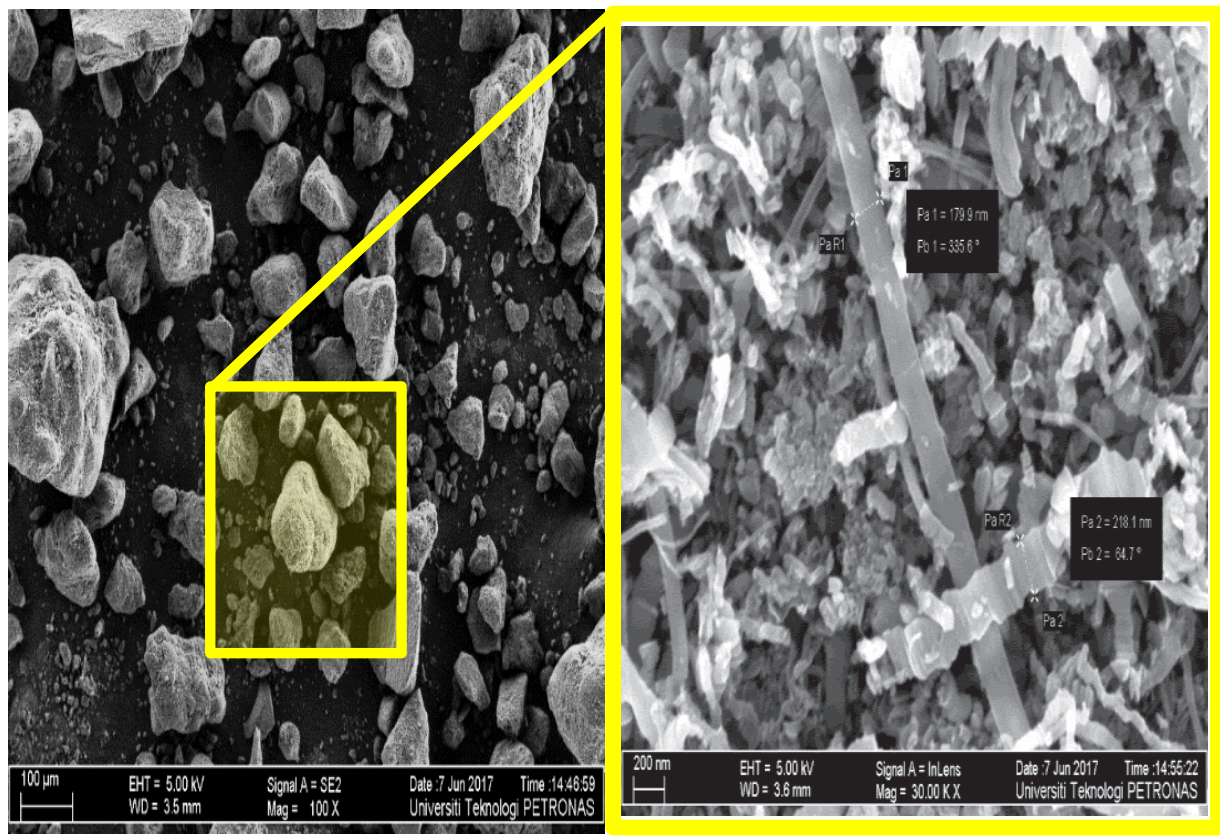

Fig. 4. FESEM of pure nano Carbon-13.

\section{Conclusion}

Integrating temperature sensor into the structure of the system enhances the system operation with greater accuracy, action, reaction, and in control capability. Equivalent preliminarily mathematical model is selected to conduct analysis of the performance of the sensor as a function of the internal energy, the thermodynamic volume displacement and the number of partials of the system were also involved. Thus, carbon-13 material has been carefully chosen to develop a remotely precise temperature sensor that is suitable for many applications. This paper focuses on analysis of temperate in nanoscale system via statistical thermodynamics approach along with the characterization of carbon-13 by Powder X-ray Diffractometer, X-Ray Photoelectron Spectrometer and Variable pressure field emission scanning electron microscope.

The author acknowledges the motivation of Universiti Teknologi PETRONAS, Malaysia, for this research work. Also, Ministry of Higher Education in Malaysia (MOHE) is acknowledged for supporting this research under grant number [0153AA-B91].

\section{References}


1. J. f. Stebbins, modern methods in solid-state NMR A Practitioner's Guide, RSC Adv., Stanford CA, (2017)

2. C. Tropea, A. L. Yarin, Springer handbook of experimental fluid mechanics, Darmstadt Germany, (2007)

3. V. Ingegnoli, Thermodynamics-Systems in Equilibrium and Non-Equilibrium, Croatia (2011)

4. M. A. Abdullah, T. M. B. Albarody, P. S. M. B. M. Yusoff, MATEC 131, 01008 (2017)

5. M. Vladimirova, S. Cronenberger, D. Scalbert, I. Ryzhov, V. Zapasskii, G. Kozlov, Phys. Rev. B: Condens. Matter 97, 041301 (2018)

6. G. J. Beran, J. D. Hartman, Y. N. Heit, Am. Chem. Soc. 49, 2501 (2016)

7. K. Kanehashi, Nippon Steel Tech. Report, Adv. Technology Research Lab., (2011)

8. J. F. Stebbins, Chem. Rev. 7, 1353 (1991)

9. P.W. Ma, S. Dudarev, A. Semenov, C. Woo, Phys. Rev. E: Stat. Phys., Plasmas, Fluids 82, 031111 (2010)

10. S. Coles, J. Bawa, L. Trenner, P. Dorazio, An introduction to statistical modeling of extreme values, Stuart UK (2001)

11. T. S. Biró, Is there a temperature?: conceptual challenges at high energy, acceleration and complexity, Heidelberg UK (2011)

12. Y. N. Kaznessis, Statistical thermodynamics and stochastic kinetics: An introduction for engineers, UK (2011)

13. N. Dalarsson, M. Dalarsson, and L. Golubovic, Introductory statistical thermodynamics, Morgantown USA (2011)

14. M. J. Hoch, Statistical and thermal physics: an introduction, Tallahassee USA (2011)

15. C. Nanotubes, Top. Appl. Phys. 111, 353 (2008)

16. C. Schorn, NMR Spectroscopy: Data Acquisition, New York USA (2002)

17. R. S. Macomber, A Complete Introduction to Modern NMR Spectroscopy, USA (1998)

18. W. V. Eberhard Breitmaier, Carbon-13 NMR Spectroscopy, Germany (1989)

19. S. S. Han, NMR Imaging in Chemical Engineering, Germany (2006)

20. A. U. Ahmad, ${ }^{13} C-N M R$ of Natural Products, New York USA (1992)

21. J. Fayos, S. State Chem. 148, 278 (1999)

22. A. Gibaud, A. Bap., D. Dos., C. Bri., L. Ya., B. Ock, Europhys. Lett. 63, 833 (2003)

23. S. O. Pillai, Applied Physics As Per Jntu Syllabus, (2006)

24. T. Okpalugo, P. Papakon., H. Mur., J. McLaugh., N. Brown, Carbon 43, 153 ( 2005)

25. A. Alyamani, O. Lemine, Ed. InTech 23, 473 (2012) 